\title{
Correction: Hepatic gene expression explains primary drug toxicity in bipolar disorder
}

Anna Maria Birkl-Toeglhofer, Christoph Birkl, Ida Cirila Llenos, Serge Weis and Johannes Haybaeck

\section{Correction to: Translational Psychiatry \\ https://doi.org/10.1038/s41398-019-0666-4 \\ published online 9 December 2019}

The original Article had an incomplete acknowledgements list. The following sentence has now been added to the HTML and PDF versions of this Article:
"This study was performed within the framework of the $\mathrm{PhD}$ program Molecular Medicine of the Medical University of Graz. This work was supported by the Medical University of Graz within the Open Access Publishing Funding Program."

Published online: 17 February 2020 